\title{
Terapia com Raloxifeno na Pós-menopausa: Efeitos sobre o Sistema Hemostático
}

Autor: George Dantas de Azevedo

Orientador: Prof. Dr. Marcos Felipe Silva de Sá

Co-Orientador: Prof. Dr. Rendrik França Franco

Tese apresentada à Faculdade de Medicina de Ribeirão Preto da Universidade de São Paulo, para obtenção do título de Doutor em Medicina, Área de concentração: Tocoginecologia, em 29 de outubro de 2001.

O cloridrato de raloxifeno é um derivado benzotiofeno não esteróide, da classe dos moduladores seletivos do receptor estrogênico, que exerce efeitos tecido-específicos, produzindo agonismo estrogênico sobre o tecido ósseo e perfil lipídico, mas sem atividade estimulatória para as mamas e útero. Semelhante à terapia com estrogênios, o uso de raloxifeno está associado com aumento de três vezes do risco de tromboembolismo venoso, entretanto, os mecanismos que contribuem para esse risco aumentado e os efeitos dessa droga sobre o sistema hemostático não têm sido amplamente relatados.

Objetivo: analisar os efeitos do raloxifeno sobre o sistema hemostático, em mulheres na pós-menopausa. Pacientes e Métodos: foram estudadas 20 mulheres saudáveis na pós-menopausa, com idade de 56,8 \pm 5,9 anos (média \pm desvio-padrão), sendo que 16 delas concluíram o estudo. As pacientes foram tratadas com cloridrato de raloxifeno (60 mg por dia), durante $6 \mathrm{me}-$ ses. Foram realizadas avaliações laboratoriais do sistema hemostático no período pré-tratamento e após transcorridos 1, 3 e 6 meses de uso da medicação. Parâmetros considerados: atividades plasmáticas dos diversos fatores da coagulação (II, V, VII, VIII, IX, X, $\mathrm{XI}$, XII e fibrinogênio), antitrombina, proteína $\mathrm{C}$, níveis de proteína $S$ total, niveis de fragmento $1+2$ da protrombina, sensibilidade à proteína $\mathrm{C}$ ativada e provas de agregação plaquetária. Análise estatística: a verificação da normalidade dos valores amostrais foi realizada utilizando-se o teste de Kolmogorov-Smirnov. Para as comparações dos resultados obtidos nas diversas fases da pesquisa foram utilizados o teste ANOVA para medidas repetidas e o teste de NewmanKeuls. Para a comparação de dados entre dois períodos específicos de tratamento, foi utilizado o teste " $\mathrm{t}$ " pareado. O teste não-paramétrico de Friedman foi usado para analisar os resultados de agregação plaquetária. Em todos os procedimentos estatísticos foi empregado nivel de significância de $5 \%$.

Resultados: o tratamento com raloxifeno causou aumento dos níveis plasmáticos dos fatores VIII, XI e XII da coagulação $(\mathrm{p}<0.05)$. A atividade do fator VIII aumentou de $17,1 \%$ e $27 \%$, após 3 e 6 meses de tratamento, respectivamente, em comparação aos valores basais. A atividade do fator XI aumentou 11,1\%, após 6 meses de tratamento com raloxifeno. Quanto ao fator XII, observamos aumentos significativos de 34,6\%, $49,5 \%$ e 49,3\%, após 1,3 e 6 meses de uso do raloxifeno, respectivamente. Foram evidenciadas reduções significativas dos niveis de antitrombina, após $3(-5,1 \%)$ e 6 meses $(-6,5 \%)$ de tratamento, e dos níveis de fibrinogênio $(-6,4 \%)$, após 1 mês. Ocorreu redução significativa da sensibilidade à proteína $\mathrm{C}$ ativada, após 6 meses de uso do raloxifeno, em comparação ao período pré-tratamento. O tratamento não afetou de forma significativa os niveis médios de fragmento $1+2$ da protrombina. Também não foram observadas diferenças estatisticamente significativas nos valores máximos de agregação plaquetária, entre os períodos estudados.

Conclusões: os resultados desse estudo prospectivo sugerem que o risco aumentado de tromboembolismo venoso associado à terapia com raloxifeno poderia ser explicado, pelo menos em parte, por um estado prócoagulante relacionado a aumentos das atividades coagulantes dos fatores VIII e XI, diminuição da atividade anticoagulante da antitrombina e redução da sensibilidade à proteína $\mathrm{C}$ ativada.

Palavras-chave: Menopausa. SERM. Hemostasia. Agregação plaquetária. Tromboembolismo.

\section{Efeitos da Metiltestosterona sobre a Sexualidade, Metabolismo Lipoproteíco e Hepático e Niveis de Testosterona em Mulheres na Pós-Menopausa em Uso de Reposição Estroprogestativa}

Autor : Fernando José Felipe de Paula Orientador: Prof. Dr. Mauro Abi Haidar Co-orientador: Prof. Dr. Edmund Chada Baracat

Dissertação apresentada na Escola Paulista de Medicina - Universidade Federal de São Paulo, em 3 maio de 2001 para obtenção de Título de Mestre.

Objetivos: Avaliar os efeitos da metiltestosterona, na dose diária de 2,5 mg, sobre a sexualidade de mulhe- res pós-menopáusicas em uso de reposição estroprogestativa, além de estudar seus efeitos sobre o 
colesterol total e frações, enzimas hepáticas (TGO/ TGP) e niveis hormonais (testosterona total e livre). Casuística e métodos: Estudamos 40 pacientes divididas em quatro grupos. O estudo é duplo cego placebo controlado do tipo crossover (inversão do tratamento para os grupos 3 e 4).Comparamos a média de escore de cada grupo, obtida por questionário validado, em cada fase do estudo. As variáveis avaliadas foram prazer sexual, fantasias e excitação, freqüência de relações e dor ao coito. Também comparamos os valores médios de todos os exames colhidos de cada grupo em cada fase.

Resultados: A média de escore das respostas obtidas com o grupo 1 (placebo) não sofreu alteração durante todo o estudo em relação às variáveis estudadas. No grupo 2 (metiltestosterona), a média de escore elevouse significativamente para as variáveis prazer e fantasia sexual. No grupo 3, durante a fase em que usou placebo não houve alterações, porém quando passou a usar metiltestosterona constatou-se elevação significativa no escore das respostas relacionadas a prazer e fantasias sexuais. O inverso ocorreu com o grupo 4, no qual inicialmente notou-se melhora das respostas seguido por piora significativa. Não houve alterações no escore médio das demais variáveis estudadas entre os grupos. Valores médios de HDL-colesterol diminuíram com a metiltestosterona em comparação ao placebo, ao passo que os valores médios de testosterona total e livre elevaram-se, ambos significativamente. Os demais exames avaliados não apresentaram diferenças entre os grupos.

Conclusões: Metiltestosterona 2,5mg ao dia melhorou, de forma significativa, as respostas relacionadas ao prazer sexual, fantasia e excitação, sem interferir na freqüência de relações. Reduziu niveis de HDLcolesterol, aumentou os de testosterona total e livre sem provocar efeitos arrenomiméticos. Não interferiu nos demais exames avaliados.

Palavras Chave: Pós-menopausa. Sexualidade. Androgênios.

RBCO 24(1):68,2002

Resumos de Tese

\section{Efeitos do Tabagismo na Circulação Arterial Materna e Fetal: Estudo Através do Método Doppler}

Autor: Renata Alberge Giugliano Meschino

Orientador: Prof. Dr. Francisco Mauad Filho

Dissertação de Mestrado apresentada à Faculdade de Medicina de Ribeirão Preto da Universidade de São Paulo, em 4 de junho de 2001.

Realizou-se estudo prospectivo e comparativo para avaliar as repercussões hemodinâmicas do tabagismo no binômio materno-fetal. O estudo compreendeu a análise dos Índices de Resistência (IR) e Índice de Pulsatilidade (IP) dos vasos arteriais maternos (artérias uterinas) e fetais (artérias umbilical, aorta abdominal e artéria cerebral média), utilizando-se aparelho de Doppler pulsátil colorido, mensurando-se também a freqüência cardíaca materna e fetal. Procedeuse ao exame em quarenta gestantes da rede municipal de saúde de Ribeirão Preto e região, atendidas na Escola de Ultra-Sonografia e Reciclagem Médica Ribeirão Preto, em colaboração com o Departamento de Ginecologia e Obstetrícia da Faculdade de Medicina de Ribeirão Preto. O grupo controle (GC), foi constituído de vinte gestantes normais sem intercorrências clínicas e obstétricas na gravidez, e o grupo tabagista (GT) composto por vinte gestantes tabagistas crônicas fumantes de dez ou mais cigarros ao dia. No GT o exame era realizado em duas etapas: antes (GTA) e após (GTD) o consumo de dois cigarros, contendo um total de 1,8 $\mathrm{mg}$ de nicotina e $26 \mathrm{mg}$ de monóxido de carbono. Para avaliar os efeitos crônicos do tabagismo, comparou-se o GC com o GTA aplicando-se o teste de Mann-Whitney. Comparando-se o GTA com o GTD, verificou-se a ação aguda do cigarro utilizando-se o teste de Wilcoxon. Como resultados significativos, foi possivel identificar um aumento da vascularização cerebral dos fetos de mães tabagistas em relação aos fetos do GC, observado através de uma diminuição significativa do IP. Confrontando-se GTA com GC, verificou-se ainda uma eleção do IR da artéria uterina direita, aumento da velocidade diastólica das artérias umbilicais, e diminuição da freqüência cardíaca materna no GTA. Os efeitos agudos após o consumo de dois cigarros não foram significativos em nenhuma da variáveis estudadas. Os resultados desta pesquisa sugerem que os fetos de mães tabagistas crônicas apresentam um vasodilatação cerebral como provável defesa à hipóxia fetal crônica; entretanto, após o consumo de dois cigarros, não há efeitos agudos na hemodinâmica materna e fetal com relação aos vasos estudados.

Palavras-Chave: Dopplervelocimetria. Tabagismo. Gravidez normal. Droga na gravidez. 


\section{Avaliação da Função Ovariana Pós-histerectomia Total Abdominal em Mulheres no Menacme}

Autor: Eliana Aguiar Petri Nahás

Orientadora: Profa. Dra. Anaglória Pontes

Tese apresentada ao curso de Pós-Graduação em Ginecologia e Obstetrícia, área de concentração em Ginecologia, da Faculdade de Medicina de Botucatu - UNESP, para obtenção do Título de Doutor, em 28 de novembro de 2001.

Objetivo: Avaliar a função ovariana, em mulheres no menacme submetidas à histerectomia total abdominal (HTA), por meio de parâmetros clínicos, endocrinológicos e ultra-sonográficos.

Pacientes e Métodos: Estudaram-se, prospectivamente, 61 mulheres, com 40 anos ou menos em 3 grupos: G1, composto por 31 pacientes submetidas à HTA e, G2, por 30 mulheres normais. Critérios de inclusão: eumenorréicas, ovulatórias, não obesas ou fumantes, sem cirurgias prévias, sem uso de medicações ou de endocrinopatias, com FSH basal < $15 \mathrm{mIU} / \mathrm{ml}$ (duas ocasiões). Realizaram-se dosagens de FSH, LH, estradiol $\left(\mathrm{E}_{2}\right)$ e inibina B nos momentos basal, aos 2, 6 e 12 meses. O volume ovariano na ultra-sonografia (US), o Índice de Pulsatilidade (IP) das artérias ovarianas pela dopplervelocimetria e o Valor de Maturação (VM) na colpocitologia foram mensurados inicialmente e aos 6 e 12 meses.

Resultados: Na comparação estatística inicial, os grupos foram homogêneos. Nas pacientes submetidas à histerectomia, aos 6 e 12 meses, ocorreu aumento do
$\mathrm{E}_{2}$ e redução da inibina $\mathrm{B}(\mathrm{p}<0,05)$, sem alterarção dos níveis de FSH e LH. Ao US, observou-se aumento do volume ovariano e diminuição do IP ao Doppler $(p<0,05)$, quando confrontado ao controle. Aos 12 meses houve redução do VM $(\mathrm{p}<0,05)$ no $\mathrm{G} 1$. Ao final do estudo, apenas entre as pacientes histerectomizadas, observouse que $12,9 \%$ (4/31) apresentaram $\mathrm{FSH}>40 \mathrm{mIU} / \mathrm{ml}$, estradiol $<20 \mathrm{pg} / \mathrm{ml}$ e inibina $\mathrm{B}<5 \mathrm{ng} / \mathrm{ml}$, valores compativeis com falência ovariana. No grupo controle não houve alteração de nenhum desses parâmetros. Conclusão: Nas pacientes submetidas à HTA ocorreu redução significativa da inibina $\mathrm{B}$, sem alterações de FSH e LH. Houve aumento do volume ovariano e redução do indice de pulsatilidade, ao doppler. Esses resultados sugerem que, a histerectomia total abdominal acelera o declínio da função ovariana em mulheres no menacme.

Palavras-chave: Função ovariana. Histerectomia. Dopplervelocimetria. Falência ovariana.

\section{Linfonodo Sentinela no Carcinoma Infilltrativo Inicial de Mama: Estudo de sua Localização e de sua Capacidade Preditiva em Relação ao Estado da Axila}

Autor: José Roberto Morales Piato

Orientador: Prof. Dr. Alfredo Carlos S.D. de Barros

Tese apresentada à Faculdade de Medicina da Universidade de São Paulo para a obtenção do título de Doutor em Medicina, em 27 de maio de 2001.

Introdução: Existe comprovação de que na maioria das pacientes com carcinoma inicial de mama os linfonodos axilares apresentam-se livres de metástases. Tal fato tem motivado a pesquisa de método capaz de predizer o estado da axila, com vista a evitar esvaziamentos axilares desnecessários.

Objetivo: Avaliar a capacidade do estudo do linfonodo sentinela (LS), por técnica de medicina nuclear, para a predição do estado linfonodal axilar em portadoras de carcinoma inicial de mama (T1-T2 e NO) submetidas ou não a quimioterapia neoadjuvante.

Método: Foram selecionadas 86 pacientes, sendo 44 que não receberam qualquer medicação prévia (grupo I) e 42 que haviam sido submetidas a quimioterapia neoadjuvante, com três ciclos do esquema adriamicina e ciclofosfamida (grupo II). No dia anterior à cirurgia procedeu-se injeção peritumoral de $0,2 \mathrm{~mL}$ de dextran 70 , marcado por ${ }^{99 \mathrm{~m}} \mathrm{Tc}$ com atividade de $15 \mathrm{MBq}$, que foi orientada por estereotaxia nas lesões não palpáveis. A localização pré-operatória do LS foi obtida através de linfocintilografia, utilizando-se gama-câmara convencional. No momento da cirurgia empregou-se detector de radiação gama (probe). A radiação captada, que se traduziu em sinais acústicos, orientou a biópsia do LS. Superficies de cortes dos linfonodos identificados pelo probe, obtidas a cada $3 \mathrm{~mm}$, foram examinadas intra-operatoriamente por técnica de imprint. Em seguida realizou-se dissecção axilar completa e exame histopatológico definitivo do LS por cortes seriados a cada 50 ?m e dos demais linfonodos da axila por técnica convencional.

Resultados: Obtivemos identificação do LS em 83 pacientes (96,5\%). Em duas pacientes do grupo I e em uma do grupo II, todas elas com lesões não palpáveis, não foi possivel obter captação do radiofármaco. Em relação ao grupo I, em nenhuma paciente ocorreu resultado falso-negativo, enquanto que esta falha ocorreu em três pacientes $(17 \%)$ do grupo II.

Conclusão: Os índices de acurácia do método foram de $100 \%$ para as pacientes sem medicação prévia e 93\% para aquelas submetidas a quimioterapia neoadjuvante.

Palavras-chave: Câncer de mama. Quimioterapia. Linfonodo sentinela. 


\title{
Prevalência das Neoplasias Intra-epiteliais Cervicais e Lesões Induzidas pelo HPV nas Mulheres Soropositivas/AIDS
}

\author{
Autora: Susana Cristina Aidé Viviani Fialho \\ Orientador: Prof. Dr. Gutemberg Leão de Almeida Filho
}

Dissertação de Mestrado apresentada ao Instituto de Ginecologia da Universidade Federal do Rio de Janeiro, em 13 de dezembro de 2000.

Através de estudo transversal descritivo de uma série de casos, foram analisadas 130 mulheres infectadas pelo HIV sob o aspecto epidemiológico e por meio da citopatologia, colposcopia e biópsia dirigida. A idade média das pacientes foi de 33,1 anos e 40,2\% delas fumavam. A via sexual foi a mais freqüente na transmissão do HIV $(97,7 \%)$ as não-usuárias de drogas constituíram a maioria (82\%). A infecção causada pelo HPV foi a principal DST (doença sexualmente transmissivel). A lesão intra-epitelial de baixo grau foi a anormalidade preponderante na citopatologia e na colposcopia. No histopatológico das biópsias do colo uterino, a NIC I (neoplasia intra-epitelial cervical grau 1) foi o achado mais freqüente. O mesmo exame para a vagina, a vulva e a região perianal apresentou predominância do HPV com $64,9 \%, 87,2 \%$ e $81,1 \%$, respectivamente. Houve concordância moderada entre a citopatologia e a histopatologia das biópsias do colo uterino. A preva- lência da NIC foi de 30\%; da NIVA (neoplasia intraepitelial vaginal), 5,4\%; da NIV (neoplasia intra-epitelial vulvar), $2,3 \%$ e da NIPA (neoplasia intra-epitelial perianal), 1,5\%. A prevalência das lesões HPV induzidas foi de $8,5 \%$ no colo, $18,5 \%$ na vagina, $31,5 \%$ na vulva e $10 \%$ na região perianal. O autor concluiu que a prevalência da NIC foi elevada nas mulheres HIV/AIDS e a NIC I foi o mais freqüente achado cito/histopatológico; o principal sítio das neoplasias intra-epiteliais foi o colo uterino e o das lesões HPV induzidas, a vulva, ambas tendendo à unicentricidade. A citopatologia deve ser utilizada como método de rastreio nas mulheres infectadas pelo HIV.

Palavras-chave: Aids. HPV. Doença sexualmente transmissivel. NIC. Colo: lesões pré-neoplásicas. Vulva.

\section{Avaliação do Potencial de Peroxidação Lipídica no Fluido Peritoneal de Mulheres Inférteis com Endometriose Pélvica}

Autora: Vivian Ferreira do Amaral

Orientador: Prof. Dr. Rui Alberto Ferriani

Tese de Doutorado apresentada à Faculdade de Medicina de Ribeirão Preto da Universidade de São Paulo, em 21 de maio de 2001. Área de concentração: Tocoginecologia.

Objetivo: a meta do presente estudo foi avaliar a capacidade de peroxidação lipidica no fluido peritoneal de mulheres inférteis com endometriose pélvica e mulheres normais.

Casuistica: e métodos: foram selecionados prospectivamente 32 mulheres, distribuidas em dois grupos, dispostos em grupo endometriose, composto por 17 mulheres inférteis com endometriose pélvica, comprovada por laparoscopia e biópsia, e grupo controle, determinado por 15 mulheres sem endometriose pélvica, comprovada por laparoscopia e submetidas à laqueadura tubária bilateral. A peroxidação lipidica foi avaliada através da peroxidação de TBARS (substâncias reativas ao ácido tiobarbitúrico), determinados pelo malondialdeído (MDA), malondialdeído com adição de cobre $\left(\mathrm{MDA} / \mathrm{Cu}^{2+}\right.$ ) e pleo Colest-3,5-dieno-7-7ona (CSD), expressos em $\mathrm{nmol} / \mathrm{mL}$ e $\mathrm{mg} / \mathrm{mL}$, respectivamente. As amostras de fluido peritoneal foram colhidas durante a laparoscopia, na fase folicular do ciclo menstrual. Resultados: não foram encontradas diferenças estatísticamente nos niveis de MDA entre os grupos endometriose (mediana de $0,07 \mathrm{nmol} / \mathrm{mL}$ ) e controle (mediana de $0,04 \mathrm{nmol} / \mathrm{mL})(\mathrm{p}=0,09)$. Após a peroxidação provocada pelo cobre também não houve diferença entre os grupos endometriose $(0,34 \mathrm{nmol} / \mathrm{mL})$ e controle $(0,21 \mathrm{nmol} / \mathrm{mL})$, mas houve elevação intragrupo $(\mathrm{p}<0,01)$. Diferenças estatísticas dos niveis de CSD nos Grupos endometriose e controle (mediana de 0,24 e $0,25 \mathrm{mg} / \mathrm{mL})$ respectivamente $(\mathrm{p}=0,50)$ não foram encontradas.

Conclusão: nosso estudo não demonstrou aumento do potencial de peroxidação lipídica no fluido peritoneal de mulheres inférteis com endometriose pélvica quando comparado a mulheres férteis.

Palavras-chave: Endometriose. Infertilidade. 


\section{Contribuição ao Estudo do Laser de Vapor de Cobre no Tratamento da Endometriose Induzida Cirurgicamente em Coelhas}

Autor: Maurício Paulo Angelo Mieli

Orientador: Prof. Dr. Paulo Roberto Bueno Pereira

Tese apresentada ao Departamento de Cirurgia da Faculdade de Medicina de São Paulo para obtenção do Título de Doutor em Medicina, em 27 de agosto de 2001.

A endometriose sempre representou um dos mais inquietantes enigmas da ginecologia, sendo motivo de vários estudos tanto para o esclarecimento de sua fisiopatologia como para a busca de novas modalidades terapêticas. Neste estudo, 42 coelhas da raça New Zealand foram submetidas a indução experimental da endometriose, de acordo com técnica cirúrgica padronizada. Após 30 dias da indução, um grupo de 29 animais foi submetido ao tratamento pelo laser de vapor de cobre (LVC) em tempos que variaram de 2, 5, 15, 30 e 60 segundos e, outro grupo de 13 coelhas, serviu como controle, tendo-se realizado apenas a laparotomia, sem que fosse realizado algum tratamento. Após 15 dias da segunda laparotomia realizou-se avaliação macroscópica da presença de aderências e estudo histopatológico quanto à presença de endometriose, glândulas, estroma, processo inflamatório agudo, processo inflamatório crônico, processo inflamatório crô- nico granulomatoso tipo corpo estranho, necrose, neoformação vascular e fibrose. O modelo experimental de indução da endometriose, realizado neste trabalho, foi adequado e de fácil reprodução, com 100\% de eficácia. O tratamento com LVC mostrou-se eficiente, com destruição da doença em 31,00 \% dos casos. Em 69,85\% das coelhas que ainda tinham a lesão, houve diminuição na quantidade de glândulas. A destruição do estroma aconteceu em $41,38 \%$ dos casos, no grupo laser. Com a energia total de 23 a $30 \mathrm{~J}$, correspondente ao tempo de 15 segundos, houve menos processo inflamatório crônico, neoformação vascular e fibrose tecidual. Os resultados sugerem que o LVC é um recurso promissor no tratamento da endometriose em seres humanos.

Palavras-chave: Laser. Endometriose. Modelo experimental.

\section{TEGO 2002 \\ Inscrições: \\ 15 de abril a 31 de maio de 2002}

INFORMAÇÕES NA

Federada do seu Estado

PROVAS:

25 de agosto de 2002 